\title{
Attenuation of Lipopolysaccharide-Induced Inflammatory Response and Phospholipids Metabolism at the Feto-Maternal Interface by $N$-Acetyl Cysteine
}

\author{
MANJEET K. PAINTLIA, AJAIB S. PAINTLIA, AVTAR K. SINGH, AND INDERJIT SINGH \\ Department of Pediatrics [M.K.P., A.S.P., I.S.]; Department of Pathology and Laboratory Medicine [A.K.S.], Medical University of South \\ Carolina, Charleston, South Carolina 29425
}

\begin{abstract}
Maternal microbial infections cause adverse fetal developmental outcomes including embryonic resorption, intrauterine fetal death, and preterm labor. Recent studies demonstrated that oxidativestress plays an important role in chorioamniotitis pathogenesis. Herein we investigated the effect of $N$-acetyl cysteine (NAC) on lipopolysaccharide (LPS)-induced preterm labor and fetal demise in murine model. Lipopolysaccharide exposure at embryonic day 18 demonstrated an increase in the abortion rate and fetal demise in pregnant rats. This was associated with increase in an inflammatory response (cytokines, chemokines, and iNOS expression) and infiltration of leukocytes (monocytes and polymorphonuclear cells) in the placenta. There was increased expression of cytosolic and secretary phospholipase A2 with increased secretion of prostaglandin-2 and leukotriene B4 in the placenta, suggestive of increased metabolism of phospholipids. In addition, expression of cycloxygenase- 2 and malondialdehyde production (oxidative-stress marker) was increased in the placenta. Conversely, NAC pretreatment abolished these effects of LPS in the placenta. Collectively, these data provide evidence that LPS-induced increased inflammation and metabolism of phospholipids at the feto-maternal interface (placenta) is critical for preterm labor and fetal demise during maternal microbial infections which could be blocked by antioxidant-based therapies. (Pediatr Res 64: 334-339, 2008)
\end{abstract}

$\mathrm{M}$ aternal microbial infections are one of the main causes of preterm labor and adverse fetal outcome, which persist as major and unresolved challenges. Pro-inflammatory cytokines are hypothesized to contribute to these adverse effects because of microbial infections. Pro-inflammatory cytokines (IL-1 $\beta$, IL-6, and TNF- $\alpha$ ) are known to stimulate arachidonic acid metabolism in the human reproductive system (1). During normal pregnancy, secretion of these cytokines from uterine tissues is important for the induction of prostaglandin production to initiate labor $(1,2)$. Whereas microbial infections induce excessive release of these cytokines

Received April 4, 2008; accepted May 15, 2008.

Correspondence: Inderjit Singh, Ph.D., Department of Pediatrics, Medical University of South Carolina, 173 Ashley Avenue, Charleston, SC 29425; e-mail: singhi@musc.edu.

This study was supported in part by grants from the National Institutes of Health: NS-22576, NS-34741, NS-37766, NS-40810, and NCRR grants C06 RR018823 and C06 RR015455 from the Extramural Research Facilities Program. in the uterus resulting in preterm labor and delivery via disproportionate release of prostaglandins $(3,4)$.

In studies exploring the mechanisms of preterm labor caused by microbial infections, lipopolysaccharide (LPS) has been often used as a bacterial pathogen model $(3,5)$. Humans are constantly exposed to low levels of LPS through microbial infections. Systemic maternal LPS exposure has been previously shown to be associated with adverse fetal developmental outcomes such as embryonic resorption, intrauterine fetal death, and preterm labor in animals (6). In addition, microbial invasion of the placenta is reported to cause chorioamniotitis and preterm labor in human patients (1). LPS is reported to induce inflammation in human gestational tissues, resulting in an increase in release of pro-inflammatory cytokines (3) and phospholipid metabolites i.e. prostanoids $(1,2)$. LPS induces the release of type II phospholipase A2 $\left(\mathrm{PLA}_{2}\right)$ in the human placenta, which promotes the formation of phospholipids metabolites (4). These LPS-induced increase in expression of pro-inflammatory cytokines and phospholipase isoenzymes in the placenta are mediated by NF- $\kappa \mathrm{B}$ activity (7).

Compounds that have antioxidant properties, such as $\mathrm{N}$-acetyl cysteine (NAC) - a glutathione precursor and direct antioxidant-have been shown to inhibit upstream events leading to $\mathrm{NF}-\kappa \mathrm{B}$ activation (8-10). NAC decreases the generation of free radicals by increasing glutathione synthesis $(8,11)$. NAC has been shown to attenuate inflammation in various disease models such as those that mimic ischemia-reperfusion injury in brain (12), lethal endotoxemia (13), and hypoxia-ischemic brain injury in neonatal brains (14). Based upon there reports, we hypothesized that maternal microbial infection induced oxidative-stress in the placenta may be responsible for preterm labor. So, in this study, we investigated the therapeutic efficacy of NAC to limit preterm labor and fetal demise in a murine model of maternal microbial infection.

Abbreviations: COX-2, cysloxygenase-2; ICAM-1, intracellular adhesion molecule-1; LPS, lipopolysaccharide; LTB4, leukotriene B4; MDA, malionaldehyde; NAC, $N$-acetyl cysteine; PLA2, phospholipase 2; VCAM-1, vascular adhesion molecule-1 


\section{MATERIALS AND METHODS}

Chemicals and reagents. LPS (Escherichia coli, serotype 055:B5), NAC, lysis buffer, and other chemicals were purchased from Sigma Chemical Co., Aldrich (Saint Louis, MO). Anti-IL-6, -COX-2, -COX-1, and -CD45 antibodies including secondary antirabbit HRP conjugated antibodies were purchased from Santa Cruz Biotechnology (Santa Cruz, CA). IL-6 and TNF- $\alpha$ detection BD OptEIA Reagent ELISA kits were purchased from BD biosciences (San Jose, CA). Prostaglandin E2 (PGE2) and leukotriene B4 (LTB4), enzymeimmunosorbant assay (EIA) kits were purchased form Assay designs Inc. (Ann Arbor, MI). Malondialdehyde (MDA) detection kits were purchased from BIOXYTECH MDA-586 (Oxis International Inc., Foster City, CA).

Animals. All experiments were performed according to the National Institutes of Health Guidelines for the Care and Use of Laboratory Animals (National Institutes of Health publication number 80-23) and were approved by the Medical University of South Carolina Animal Care and Use Committee. Animals were provided with food and water ad libitum. Timed-pregnant SD rats at embryonic day (E16) were purchased from Harlan laboratory (Harlan, IN).

Induction of preterm labor and treatments. For induction of preterm labor, an intraperitoneal injection of LPS $(0.5 \mathrm{mg} / \mathrm{kg})$ was administered to pregnant rats on gestation day E18 (15). Preterm delivery was defined by delivery of at least one pup within $48 \mathrm{~h}$ of LPS exposure. For treatment studies, an intraperitoneal injection of NAC $(50 \mathrm{mg} / \mathrm{kg})$ suspended in physiologic saline ( $\mathrm{pH}$ 7.4) was administered to pregnant rats $2 \mathrm{~h}$ before LPS administration on E18 as reported earlier (15). To evaluate the clinical relevance of NAC treatment, it was administered to pregnant rats at $0,2,4$, and $6 \mathrm{~h}$ post-LPS administration. Normal control animals treated with saline and NAC alone were also included in the study.

Collection of samples. Timing for collection of samples was at $4 \mathrm{~h}$ (E18), $24 \mathrm{~h}$ (E19), and $48 \mathrm{~h}$ (E20) post-LPS administration in each group. Briefly, pregnant rats were anesthetized with a cocktail of ketamine $(90 \mathrm{mg} / \mathrm{kg})$ and xylazine $(10 \mathrm{mg} / \mathrm{kg})$ and peritoneal cavities were exposed via a midline incision. The uterus was removed and each chorioamnion was punctured to collect amniotic fluid. Feto-placental units were removed from the uterus horns by dissociation between implantation sites. Samples were individually, snap frozen in liquid nitrogen and stored at $-80^{\circ} \mathrm{C}$. Five placentas or amniotic fluid collected from five gestational sacs/pregnant rat were pooled for various analyses mentioned below and six pregnant rats were included in each group at each time point.

Histology. Histologic studies were performed on placentas at time intervals $4 \mathrm{~h}$ (E18), $24 \mathrm{~h}$ (E19), and $48 \mathrm{~h}$ (E20) of gestation after LPS exposure. Placentas were fixed in 10\% buffered formalin (Stephens Scientific, Riverdale, NJ) and embedded in paraffin to cut $4-\mu \mathrm{m}$ thick sections. Sections were stained with anti-CD 45 antibodies using standard methods or Hoechst dye and analyzed by immunofluorescence microscopy (Olympus BX-60) with an Olympus digital camera (Optronics, Goleta, CA) as described earlier (15). The number of cells $\left(\mathrm{CD} 45^{+}\right.$or Hoechst $\left.{ }^{+}\right)$was counted by Image Pro-Plus 4.0 (Media Cybernetics, Maryland, MO) software. Sections of three placentas from six similarly treated animals in a group were examined and images of 7-9 fields/placental section were included to calculate the average number of $\mathrm{CD}_{4} 5^{+}$and $\mathrm{Hoechst}^{+}$cells in the region. Results were then plotted as the fold-change from the control values.

RNA purification and quantitative real-time-PCR (QRT-PCR) analysis. Total RNA was purified from placentas (pooled five placentas/pregnant rat) obtained from pregnant rats $(n=6)$ /group and used for cDNA synthesis using methods described earlier (16). QRT-PCR was performed using an iCycler IQ Real-Time PCR Detection System (BIO-RAD Laboratories, Hercules, CA), IQ SYBR Green Supermix (BIO-RAD Laboratories, Hercules, CA) and target gene primers (Table 1) designed and purchased from IDT Inc (Coralville, IA). Thermal cycling conditions were as follows: activation of iTaq DNA polymerase (Bio-Rad Laboratories, Hercules, CA) at $95^{\circ} \mathrm{C}$ for $10 \mathrm{~min}$, followed by 40 cycles of amplification at $95^{\circ} \mathrm{C}$ for $30 \mathrm{~s}$ and $55-60^{\circ} \mathrm{C}$ for $1 \mathrm{~min}$. Then, normalized expression data were generated by dividing the amount of target gene concentration with the amount of reference genes i.e. GAPDH or $18 \mathrm{~S}$ rRNA as described previously (16).

Enzyme-linked immunosorbent SDS sodium dodecyl sulfate assay. Amniotic fluid obtained from pregnant rats $(n=6)$ /group was used for quantification of IL-6 and TNF- $\alpha$ using BD OptEIA Reagent kits (sensitivity of assays was as $\leq 2 \mathrm{pg} / \mathrm{mL}$ of protein) as per manufacturer's instructions. Each assay was run in duplicate for each sample. Recombinant proteins of TNF- $\alpha$ and IL-6 were used as standards to calculate their levels in the samples. Plates were read on plate reader at $450 \mathrm{~nm}$.

Enzyme-immunosorbant assay. Placentas obtained from pregnant rats $(n=6)$ /group were homogenized in ethanol, centrifuged and supernatants were used for quantification of PGE2 and LTB4. Concentration of PGE2 in the supernatant was determined by using PGE2, EIA kits as per manufactur-
Table 1. List of primers forward primer $(F P)$ and reverse primer (RP) used for amplification using QRT-PCR.

\begin{tabular}{|c|c|}
\hline Gene Name & Primer \\
\hline GAPDH & $\begin{array}{l}\text { FP: } 5^{\prime} \text {-cctacccccaatgtatccgttgtg-3', } \\
\text { RP: } 5^{\prime} \text {-ggaggaatgggagttgctgttgaa-3' }\end{array}$ \\
\hline 18S rRNA & $\begin{array}{l}\text { FP: } 5^{\prime} \text {-ccagagcgaaagcatttgccaaga-3', } \\
\text { RP: 5'-tcggcatcgtttatggtcggaact-3' }\end{array}$ \\
\hline IL- $1 \beta$ & $\begin{array}{l}\text { FP: } 5^{\prime} \text {-gagagacaagcaacgacaaaatcc-3', } \\
\text { RP: } 5^{\prime} \text {-ttcccatcttcttctttgggtattg-3' }\end{array}$ \\
\hline TNF- $\alpha$ & $\begin{array}{l}\text { FP: } 5^{\prime} \text {-cttctgtctactgaacttcggggt-3', } \\
\text { RP: } 5^{\prime} \text {-tggaactgatgagagggagcc-3' }\end{array}$ \\
\hline IFN- $\gamma$ & $\begin{array}{l}\text { FP: } 5^{\prime} \text {-atttccctccccactccattag-3', } \\
\text { RP: } 5^{\prime} \text {-ctggtgacagctggtgaatca-3' }\end{array}$ \\
\hline IL-6 & $\begin{array}{l}\text { FP: } 5^{\prime} \text {-cagaaggagtggctaaggac- } 3^{\prime}, \\
\text { RP: } 5^{\prime} \text {-cactaggtttgccgagtaga- } 3^{\prime}\end{array}$ \\
\hline iNOS & $\begin{array}{l}\text { FP: } 5^{\prime} \text {-ggaagaggaacaactactgctggt- } 3^{\prime}, \\
\text { RP: } 5^{\prime} \text {-gaactgagggtacatgctggagc- } 3^{\prime}\end{array}$ \\
\hline ICAM-1 & $\begin{array}{l}\text { FP: } 5^{\prime} \text {-gtccaattcacactgaatgccacc-3', } \\
\text { RP: } 5^{\prime} \text {-ttaaacaggaactttccegccacc-3' }\end{array}$ \\
\hline VCAM-1 & $\begin{array}{l}\text { FP: } 5^{\prime} \text {-gacaccgtcattatctcctgcact-3', } \\
\text { RP: } 5^{\prime} \text {-gtgtacgagccatccacagacttt-3' }\end{array}$ \\
\hline MCP-1 & $\begin{array}{l}\text { FP: } 5^{\prime} \text {-gaccagaaccaagtgagatca-3', } \\
\text { RP: } 5^{\prime} \text {-gcttcagatttatgggtcaagt- } 3^{\prime}\end{array}$ \\
\hline CCR2 & $\begin{array}{l}\text { FP: } 5^{\prime} \text {-tctacttcttctggactccataca- } 3^{\prime}, \\
\text { RP: } 5^{\prime} \text {-ctaagtgcatgtcaaccacac-3' }\end{array}$ \\
\hline cPLA-2 & $\begin{array}{l}\text { FP: } 5^{\prime} \text {-cctgatgtggagaaggattcgaca-3', } \\
\text { RP: } 5^{\prime} \text {-ttccttggtttccctcagaacacc-3' }\end{array}$ \\
\hline sPLA2 & $\begin{array}{l}\text { FP: 5'-gccaaatctcctgctctacaaacc-3', } \\
\text { RP: } 5^{\prime} \text {-agatgtctctttcagcaactgggc-3' }\end{array}$ \\
\hline $\mathrm{COX}-2$ & $\begin{array}{l}\text { FP: } 5^{\prime} \text {-tgtcccttcgcctctttcaatgtg-3', } \\
\text { RP: } 5^{\prime} \text {-ctcttacagctcagttgaacgect-3' }\end{array}$ \\
\hline
\end{tabular}

er's manual and run in duplicate for each sample. Similarly, LTB4 was measured in the supernatants of placental tissues using LTB4, EIA kits as per manufacturer's manual and run in duplicate for each sample, and read at 405 $\mathrm{nm}$. Sensitivity of PGE2 and LTB4 assays are 13.4 and $5.63 \mathrm{pg} / \mathrm{mL}$, respectively.

Detection of lipid peroxidation. Placentas obtained from pregnant rats $(n=6)$ were homogenized in PBS, centrifuged at $10,000 \times \mathrm{g}$ for $60 \mathrm{~min}$ and supernatants were used for analysis. MDA was measured in the supernatants by using BIOXYTECH MDA-586 as per manufacturer's instructions. Plates were read at $586 \mathrm{~nm}$ and compared with MDA standards provided in the kit. Results are presented as nmol/mg protein for MDA equivalent. Protein concentration in the supernatants was measured by using Bradford's reagent.

Immunoblotting. Placentas obtained from pregnant rats $(n=6)$ were homogenized in a cell lysis buffer at $4^{\circ} \mathrm{C}$ and centrifuged. Proteins in supernatants were fractionated on $4-12 \%$ polyacrylamide-SDS gels followed by transfer of protein bands to nitrocellulose membranes. After blocking, membranes were incubated with anti-IL- 6 or -COX-2 antibodies and then with respective secondary antibodies. Immunoblots were detected using chemiluminescence's detection kits (Amersham, Arlington Heights, IL).

Data analysis. Statistical analysis was performed using GraphPad software (GraphPad Software Inc. San Diego, CA). Effects were measured by comparing with $t$ test for two data points or by analysis of variance (StudentNewman-Keuls) for multiple data points. The criterion for statistical significance was $p<0.05$.

\section{RESULTS}

NAC blocks LPS-induced preterm labor and inflammatory response in placenta. Systemic LPS injection (E18) provoked preterm labor in 10 of 14 treated pregnant rats with full emptiness of the two uterine horns within $48 \mathrm{~h}$ LPS exposure and viability of delivered pups was $60 \pm 12.7 \%$. Despite maintaining pregnancies, the remaining four rats experienced massive in utero fetal demise (72\%) and decrease in survival of pups on normal delivery day (E21) following LPS exposure (Fig. 1A). However, no maternal mortality was observed. In 

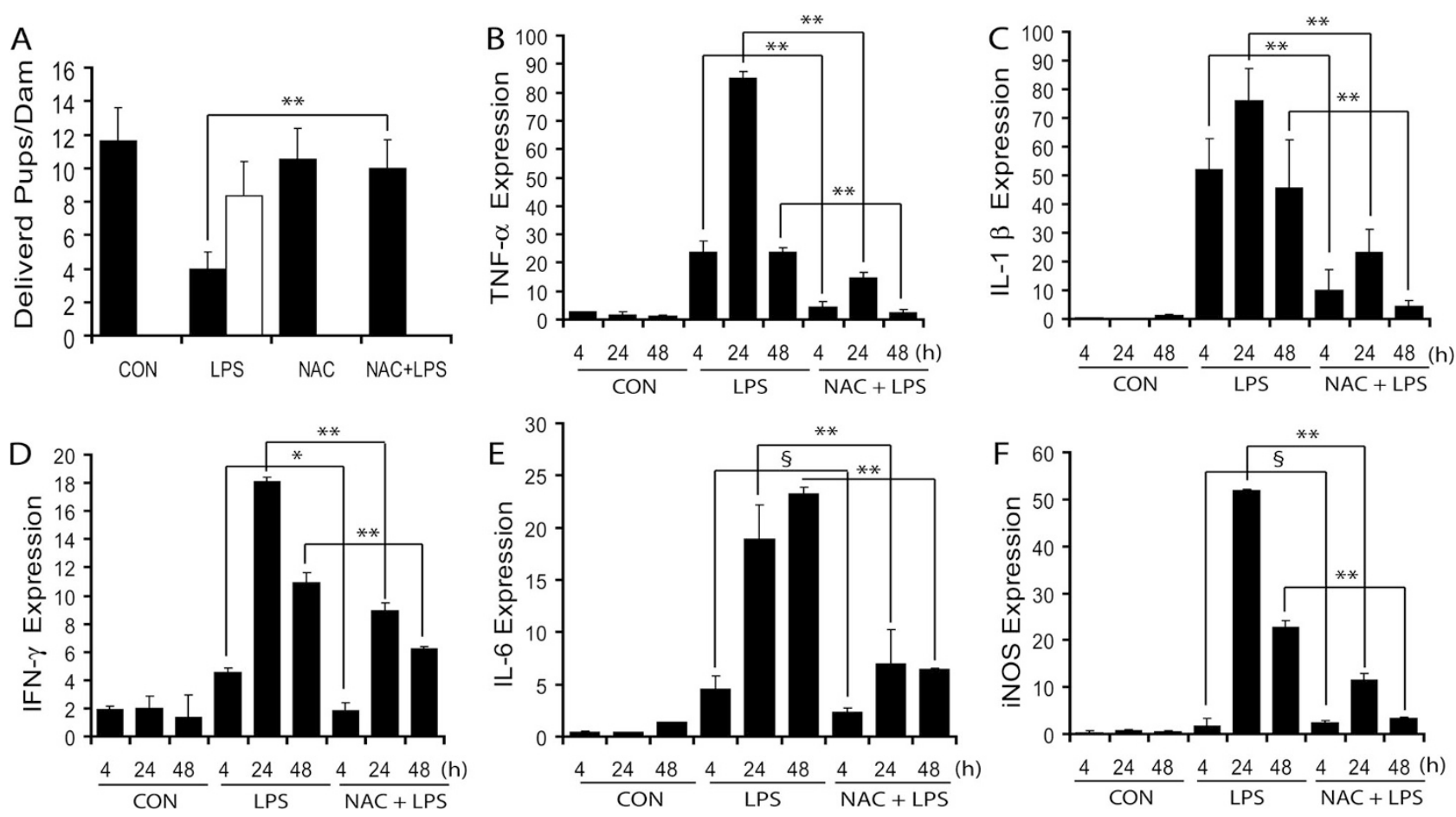

Figure 1. NAC attenuates LPS-induced fetal demise via inhibition of inflammation in the placenta of pregnant rats. Plot depicts number of live ( $\square)$ and dead pups $(\square)$ delivered by pregnant rats following LPS exposure in the presence/absence of NAC $(A)$. Plots depict transcripts of TNF- $\alpha(B)$, IL-1 $\beta(C)$, IFN- $\gamma(D)$, IL-6 $(E)$, and iNOS $(F)$ by QRT-PCR in the placentas obtained from pregnant rats $(n=6)$ following LPS exposure in the presence/absence of NAC. Results in plots are expressed as mean $\pm \mathrm{SD}$. Statistical significance ${ }^{* *} p<0.001$ and nonsignificant $(\S)$.
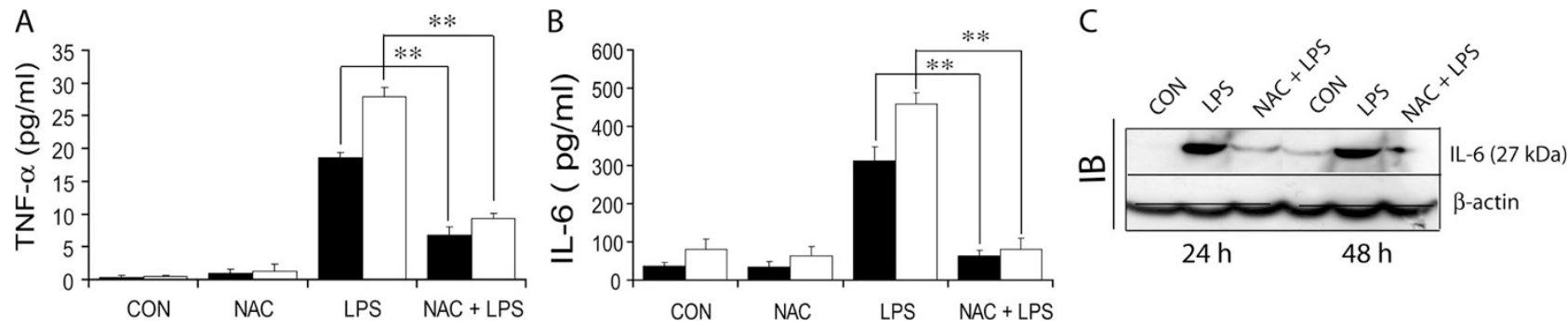

Figure 2. NAC attenuates LPS-induced inflammatory response in amniotic fluid of pregnant rats. Plots depict TNF- $\alpha(A)$ and IL-6 (B) protein in the amniotic fluid obtained form gestational sacs at $4 \mathrm{~h}(\square)$ and $24 \mathrm{~h}(\square)$ by ELISA and representative immunoblot depicts IL-6 protein in the placental tissue $(C)$ of pregnant rats $(n=6)$ following LPS exposure in the presence/absence of NAC. Results in plots are expressed as mean \pm SD. Statistical significance $* * p<0.001$ and nonsignificant $(\S)$.

contrast, NAC pretreatment significantly attenuated this LPSinduced preterm labor and fetal demise thus normal delivery (Fig. 1A). Furthermore, the evaluation of clinical relevance of NAC therapy showed that it attenuates preterm labor $100 \%$, $93 \%, 88 \%$, and $73.80 \%$ when treated at $0,2,4$, and $24 \mathrm{~h}$ following LPS exposure, respectively. QRT-PCR indicated a significant increase in LPS-induced transcripts for proinflammatory cytokines i.e., TNF- $\alpha$, IL- $1 \beta$ and IFN- $\gamma$ in the placental tissues at 4, 24 and $48 \mathrm{~h}$ postLPS exposure and this was significantly attenuated by NAC (Fig. $1 B-D$ ). Expression of these pro-inflammatory cytokines peaked at $24 \mathrm{~h}$ postLPS administration (Fig. $1 B-D$ ). Likewise, LPS induced a marked increase in IL-6 transcripts; an important pro-inflammatory cytokine at 24 and $48 \mathrm{~h}$ postLPS exposure and that was significantly blocked by NAC (Fig. 1E). In addition, iNOS transcripts produced because of inflammatory response peaked at $24 \mathrm{~h}$ post-LPS exposure and this was significantly attenuated by NAC (Fig. $1 F$ ).
NAC attenuates LPS-induced protein expression of the pro-inflammatory cytokine IL-6 and TNF- $\alpha$ in the amniotic fluid. To assess that the onset of preterm labor in LPS-exposed dams was associated with an intrauterine inflammation and that this phenomenon was prevented in the presence of NAC, we measured TNF- $\alpha$ and IL- 6 cytokines in amniotic fluids. Maternal LPS exposure at gestation E18 induced a significant increase in TNF- $\alpha$ and IL-6 in amniotic fluid at 4 and $24 \mathrm{~h}$ compared with saline treated controls (Fig. 2A, B). Conversely, NAC pretreatment attenuated the LPS-induced increase in TNF- $\alpha$ and IL- 6 at 4 and 24 h post-LPS exposure (Fig. 2A, B). Cytokines in NAC-treated control animals were similar to saline-treated controls. Corresponding with QRTPCR data (Fig. 1E), IL-6 protein was also elevated in the placental tissue following LPS exposure at 24 and $48 \mathrm{~h}$ and this was attenuated by NAC (Fig. 2C).

NAC limits LPS-induced cellular infiltration of leukocytes and chemokines expression in placenta. To further investi- 
A
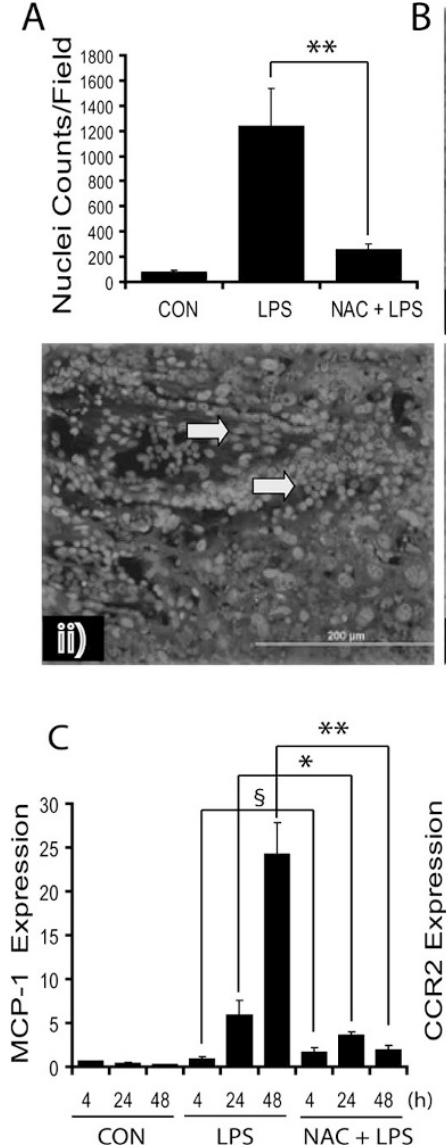

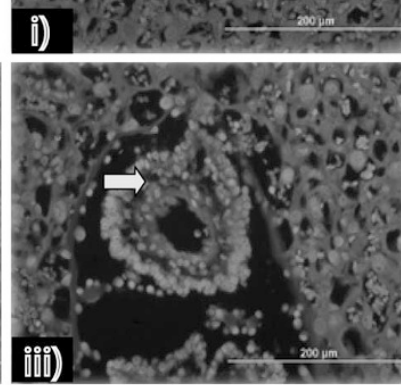

Bars; $200 \mu \mathrm{m}$ (400x)

D
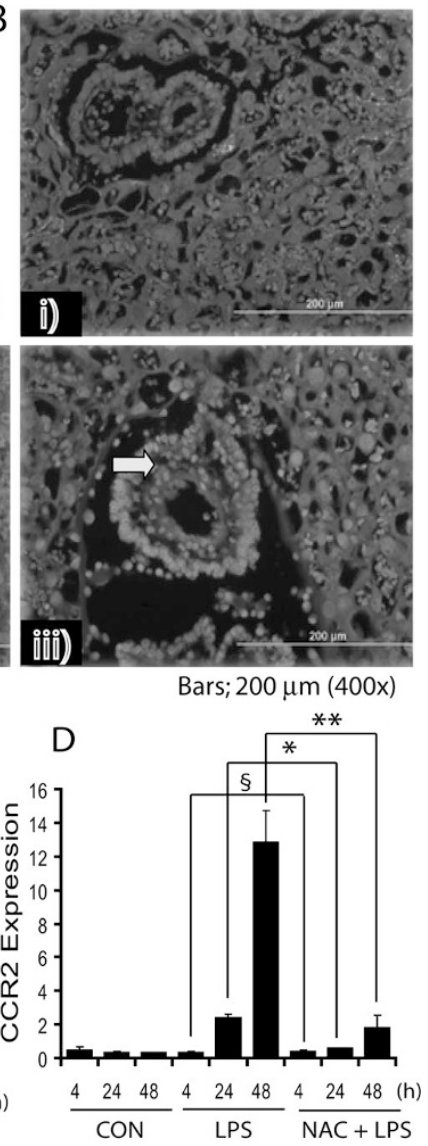

Figure 3. NAC attenuates LPS-induced leukocyte infiltration and chemokine expression in the placenta of pregnant rats. Plot depicts Hoechst ${ }^{+}$nuclei $(A)$, representative image of placental sections show cellular-infiltration [control (i), LPS (ii), and NAC + LPS (iii)] (B). Arrowhead indicates infiltrates in the placental tissue. Plots depict MCP-1 $(C)$ and CCR2 $(D)$ transcripts in the placentas of pregnant rats $(n=6)$ following LPS exposure in the presence/ absence of NAC. Results in plots are expressed as mean \pm SD. Statistical significance $* p<0.05, * * p<0.001$, and nonsignificant $(\S)$. Magnifications at $\times 400$.

gate the attenuation of LPS-induced infiltration of leukocytes and expression of chemokines in the placenta by NAC, placental tissues were analyzed for cellular infiltration and expression of MCP-1 and CCR2. MCP-1 belongs to a $\beta$-chemokine family, which is pivotal in the pathogenesis of inflammatory diseases. Hoechst staining of placental sections revealed that LPS induces infiltration of leukocytes in the placenta, which was significantly attenuated by NAC (Fig. $3 A)$. Likewise, leukocyte $\left(\mathrm{CD}_{4} 5^{+}\right)$immunostaining corroborated these data (data not shown). Histologic examination showed that NAC attenuates LPS-induced cellular infiltration into the placenta (Fig. 3B). Corroborating these results, LPSinduced increase in MCP-1 transcripts at 24 and $48 \mathrm{~h}$ after LPS exposure was significantly attenuated by NAC (Fig. 3C). Likewise, LPS induced an increase in CCR2 transcripts at 24 and $48 \mathrm{~h}$ after LPS exposure, which was significantly attenuated by NAC (Fig. 3D).

NAC attenuates LPS-induced increase in cellular adhesion molecule expression in placenta. To further characterize the effect of NAC on LPS-induced increases in expression of cell adhesion molecules (CAMs) associated with cellular in-
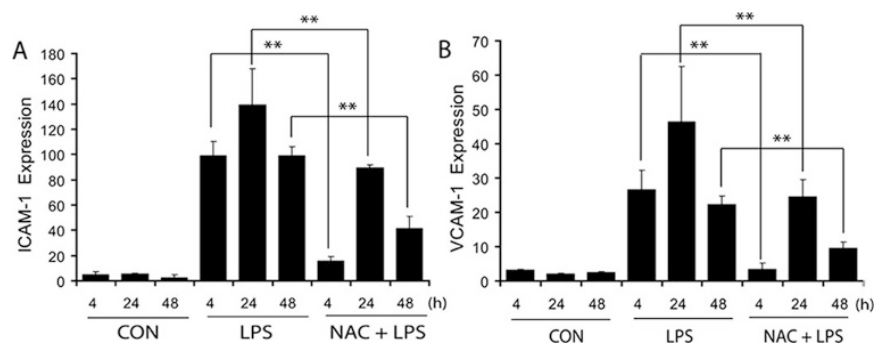

Figure 4. NAC attenuates LPS-induced expression of CAMs in the placenta of pregnant rats. Plots depict ICAM-1 $(A)$ and VCAM-1 $(B)$ transcripts in the placenta of pregnant rats $(n=6)$ following LPS exposure in the presence/ absence of NAC. Results in plots are expressed as mean \pm SD. Statistical significance $* * p<0.001$.
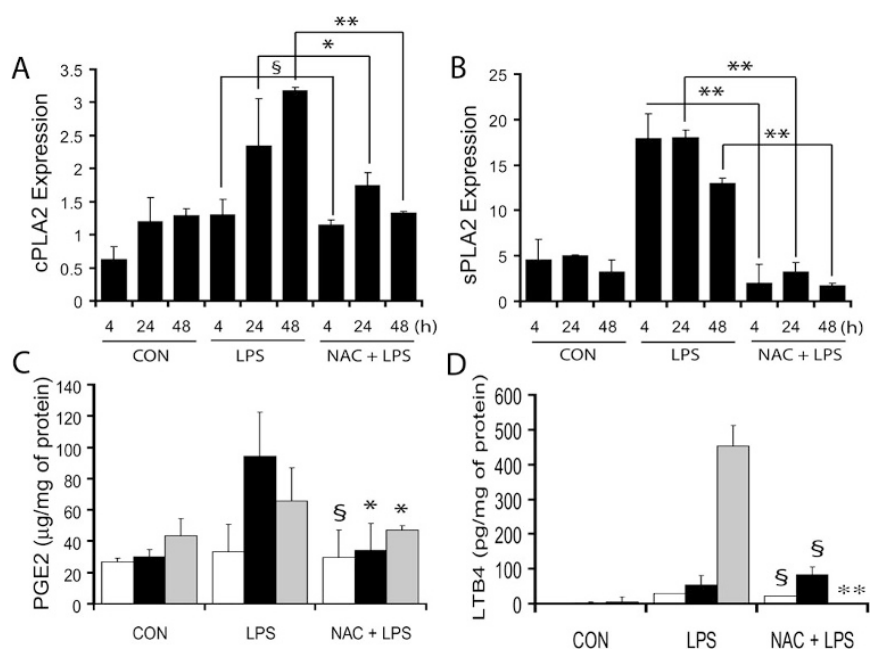

Figure 5. NAC attenuates LPS-induced phospholipids metabolism in the placenta of pregnant rats. Plots depict cPLA2 $(A)$ and sPLA2 $(B)$ transcripts in the placenta of pregnant rats $(n=6)$ following LPS in the presence/absence of NAC. Plots depict PGE2 $(C)$ and LTB4 $(D)$ level in the placentas of pregnant rats at $4 \mathrm{~h}$ (white bar), $24 \mathrm{~h}$ (black bar) and $48 \mathrm{~h}$ (gray bar) post-LPS exposure in the presence/absence of NAC. Results in plots are expressed as mean \pm SD. Statistical significance $* p<0.05$, $* *<<0.001$, and nonsignificant $(\S)$, and vs LPS for $C$ and $D$ only.

filtration, we measured critical CAMs i.e. intracellular-CAM (ICAM-1) and vascular CAM (VCAM-1) in the placental tissue. LPS induced a significant increase in ICAM-1 and VCAM-1 transcripts in the placental tissue compared with controls (Fig. 4A, B). Conversely, NAC pretreatment significantly attenuated this LPS-induced increase in ICAM-1 and VCAM-1 in placental tissues (Fig. 4A, B).

NAC inhibits LPS-induced up-regulation of phospholipids metabolism in placenta. Because inflammation increases phospholipids metabolism responsible for oxidative-stress, we next determined the level of enzymes i.e. cytoplasmic-PLA2 (cPLA2) and secretary-PLA2 (sPLA2) transcripts responsible for phospholipids metabolism and synthesis of lipid byproducts i.e. PGE2 and LTB4. LPS induced an increase in the expression of cPLA2 and sPLA2 in the placental tissue at 24 and $48 \mathrm{~h}$ following LPS exposure and this was significantly attenuated by NAC (Fig. 5A, B). Corresponding with these findings, LPS induced an increase in PGE2 in the placenta at 24 and $48 \mathrm{~h}$ after LPS exposure, which was attenuated by NAC (Fig. 5C). In addition, LPS induced an increase in LTB4 
A

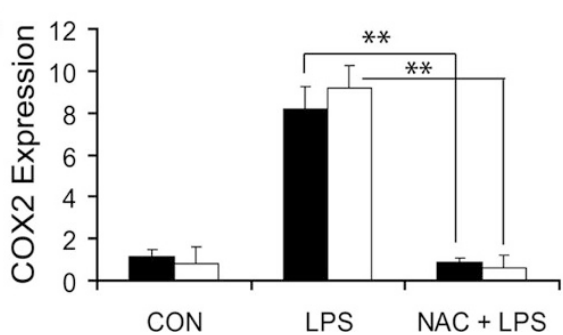

B

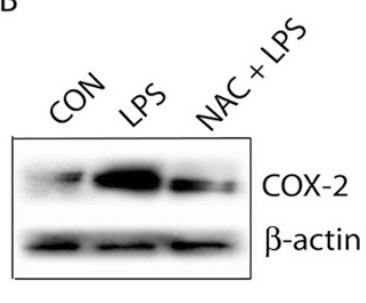

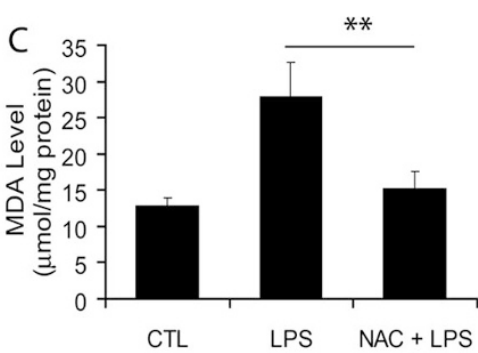

Figure 6. NAC attenuates LPS-induced phospholipids metabolism in the placenta of pregnant rats. Plot depicts COX-2 transcripts (A) at $24 \mathrm{~h}(\square)$ and $48 \mathrm{~h}$ ( $\square$ ), representative immunoblot depicts COX-2 protein $(B)$ and plot depicts MDA level $(C)$ in the placental tissues of pregnant rats $(n=6)$ following LPS exposure in the presence/absence of NAC. Results in plots are expressed as mean \pm SD. Statistical significance ${ }^{* *} p<0.001$.

at $48 \mathrm{~h}$ after LPS exposure in the placental tissue, which was attenuated by NAC (Fig. 5D).

NAC attenuates LPS-induced COX-2 expression and oxidative-stress in placenta. Because synthesis of PGE2 is associated with COX-2, we next measured COX-2 expression and generation of oxidative stress in placental tissue. LPSinduced increase in COX-2 transcripts both at 24 and $48 \mathrm{~h}$ in the placental tissue was attenuated by NAC (Fig. 6A). These data revealed that COX-2 expression is quite robust in LPSinjected pregnant rats at 24 and $48 \mathrm{~h}$ postadministration. Interestingly, NAC was quite potent in inhibiting LPS-induced COX-2 expression confirming transcriptional up-regulation of COX-2 in response to the labor-associated inflammatory milieu. Immunoblotting further confirmed that COX-2 protein $(72 \mathrm{kD})$ was also significantly elevated in the placenta at $48 \mathrm{~h}$ after LPS exposure, which was attenuated by NAC (Fig. $6 B$ ). No change in COX-1 both at mRNA and protein level was seen among the groups (data not shown). Furthermore, to determine oxidative stress in the placenta, we measured lipid peroxidation (MDA) in the placental tissue. The effects of LPS exposure at a late gestational stage is presented in Fig. $6 C$. Results indicate that LPS exposure increases MDA level in the placenta, which was significantly attenuated by NAC (Fig. $6 C)$.

Altogether, these data suggest that NAC can prevent LPSinduced preterm labor and fetal demise via attenuation of inflammation, cellular infiltration, and release of phospholipids metabolites at the feto-maternal interface of the placenta during maternal systemic/intrauterine microbial infections.

\section{DISCUSSION}

Our results indicate the therapeutic efficacy of NAC against LPS-induced preterm labor and fetal demise during maternal systemic microbial infections. LPS-induced effects in pregnant rats were mediated by inflammatory responses and oxidative stress in the placenta in addition to increase in leukocyte infiltration, CAMs expression, phospholipids metabolism, and lipid peroxidation. Conversely, LPS-induced effects in this setting were abolished by NAC.

LPS is reported to induce an increase in expression of TNF- $\alpha$ and IL- $1 \beta$ in both maternal and fetal compartments of pregnant rats when administered at E18 $(17,18)$. Clinical studies describe greater pro-inflammatory cytokines TNF- $\alpha$, IL-1 $\beta$, and IL- 6 in the amniotic fluid during intrauterine infections (19). IL-6 secretion in the fetal cavity is considered to be a determining factor of preterm labor (20). We observed a significant increase in the expression of pro-inflammatory cytokines and CAMs (ICAM-1 and VCAM-1) with corresponding increase in infiltration of leukocytes in the placenta of pregnant rats following LPS exposure compared with controls. ICAM-1 and VCAM-1 play crucial role in transmigration of leukocytes from the bloodstream to sites of inflammation. The expression of these CAMs is reported to be upregulated by pro-inflammatory cytokines (21). In addition, MCP-1 and CCR2 expression was correspondingly increased in the placenta of pregnant rats after LPS exposure compared with controls. MCP-1 activation has been associated with a majority of preterm births (22). The major function of MCP-1 is to recruit monocytes and other leukocytes to sites of inflammation. During parturition, MCP-1 may initiate or propagate labor by serving as a chemo-attractant for leukocytes in gestational tissues. It is noteworthy that MCP-1 expression is upregulated by several inflammatory mediators i.e. TNF- $\alpha$, IL- $1 \beta$ and INF- $\gamma(23)$. In addition, PGE2 has been shown to stimulate the release of MCP-1 in a placenta perfusion model (24).

In the placenta, reactive oxygen species were reported to be involved in the expression of COX-2 and iNOS in trophoblasts via activation of NF- $\kappa \mathrm{B}(25)$. COX-2 is a rate-limiting factor in prostaglandin synthesis. The contribution of COX-2 to oxidative damage has been reported to be involved in PGE2 production and hippocampal CA1 neuronal loss in a model of transient global cerebral ischemia (26). We observed that LPS induces a corresponding increase in COX-2 and PGE2 in the placenta at 24 and $48 \mathrm{~h}$ after LPS exposure to pregnant rats. This profile of change is consistent with a report describing ischemia-reperfusion ( $\mathrm{I} / \mathrm{R})$ injury induced COX-2 expression at $24 \mathrm{~h}$ after I/R injury in the placenta (27). Inflammationinduced PGE2 production is regulated by the availability of arachidonic acid and by the production of COX-2 (28). TNF- $\alpha$ has been shown to increase the synthesis of PGE2 through COX-2 activity in amniotic and decidual cells (29). Arachidonic acid is cleaved from phospholipids during metabolism by phospholipases i.e. cPLA2 and sPLA2. Similarly, we observed an increase in expression of cPLA2 and sPLA2 in the placenta after LPS exposure. PLA2 activity provides a substrate for peroxidase, COX-2, and PGE synthase responsible for synthesis of PGE2. LPS has been shown to cause the depletion of intracellular glutathione and an increase in MDA, a byproduct of lipid peroxidation (30). An observed increase in MDA in the placental tissue is suggestive of LPS-induced ROS 
generation in the placenta of pregnant rats. Lipid peroxidation and a decrease in antioxidant mechanisms are reported to be responsible for damage in patients who miscarry (31).

A popular therapeutic approach for the attenuation of LPSinduced ROS generation and lipid peroxidation-mediated preterm labor and fetal demise is the use of antioxidants. NAC is a widely investigated agent with both antioxidant and antiinflammatory properties. We and others have previously shown that NAC attenuates brain white matter injury in a systemic maternal infection model of periventricular leukomalacia $(15,32)$. NAC induced beneficial effects are attributed to free radical quenching via its direct antioxidant properties and synthesis of intracellular reduced GSH. In addition, antiinflammatory activities of NAC are attributed to suppression of pro-inflammatory cytokine expression/release (33), inhibition of adhesion molecule expression (34), and inhibition of $\mathrm{NF}-\kappa \mathrm{B}$ activation (10).

Overall, this study illustrates the underlying mechanism of preterm labor resulting from oxidative stress, increased lipid peroxidation, and metabolism of phospholipids across the feto-maternal interface via activation of the arachidonic acidcyclooxygenase pathway. It is likely that pro-inflammatory cytokines induce up-regulation of prostaglandin production in gestational tissues contributing to the preterm labor during microbial infections. Based upon these findings, we suggest that NAC may be evaluated for its therapeutic efficacy in the management and limitation of preterm labor in pregnant rats during systematic microbial infections.

In summary, we conclude that LPS-induced inflammatory response induces leukocyte infiltration, expression of CAMs and chemokines, phospholipids metabolism, and lipid peroxidation in the placenta of pregnant rats resulting in preterm labor and fetal demise. In contrast, a single injection of NAC blocks these LPS-induced adverse effects in pregnant mothers.

Acknowledgments. We thank all members of our laboratory for their valuable comments and help during the course of this study. We thank especially Dr. Shalendra Giri, PhD, for detection of PGE2 and LTB4 in the placental tissue and Dr. Jennifer G. Schnellmann, $\mathrm{PhD}$, for critical reading of this article.

\section{REFERENCES}

1. Mitchell MD, Edwin SS, Lundin-Schiller S, Silver RM, Smotkin D, Trautman MS 1993 Mechanism of interleukin-1 beta stimulation of human amnion prostaglandin biosynthesis: mediation via a novel inducible cyclooxygenase. Placenta 14:615-625

2. Dowling DD, Romero RJ, Mitchell MD, Lundin-Schiller S 1991 Isolation of multiple substances in amniotic fluid that regulate amnion prostaglandin E2 production: the effects of gestational age and labor. Prostaglandins Leukot Essent Fatty Acids 44:253-255

3. Fortunato SJ, Menon RP, Swan KF, Menon R 1996 Inflammatory cytokine (interleukins 1, 6 and 8 and tumor necrosis factor-alpha) release from cultured human fetal membranes in response to endotoxic lipopolysaccharide mirrors amniotic fluid concentrations. Am J Obstet Gynecol 174:1855-1861; discussion 1861-1862

4. Farrugia W, Nicholls L, Rice GE 1999 Effect of bacterial endotoxin on the in vitro release of Type II phospholipase-A2 and prostaglandin E2 from human placenta. J Endocrinol 160:291-296

5. Anteby EY, Johnson RD, Huang X, Dryden DK, Nelson DM, Sadovsky Y 1998 Lipopolysaccharide enhances the transcription of prostaglandin H synthase-2 gene in primary human trophoblasts. Am J Obstet Gynecol 178:469-473

6. O'Sullivan AM, Dore CJ, Boyle S, Coid CR, Johnson AP 1988 The effect of campylobacter lipopolysaccharide on fetal development in the mouse. J Med Microbiol 26:101-105

7. Lappas M, Permezel M, Georgiou HM, Rice GE 2004 Regulation of phospholipase isozymes by nuclear factor-kappaB in human gestational tissues in vitro. J Clin Endocrinol Metab 89:2365-2372
8. Neuschwander-Tetri BA, Bellezzo JM, Britton RS, Bacon BR, Fox ES 1996 Thiol regulation of endotoxin-induced release of tumour necrosis factor alpha from isolated rat Kupffer cells. Biochem J 320:1005-1010

9. Verhasselt V, Vanden Berghe W, Vanderheyde N, Willems F, Haegeman G, Goldman M $1999 \mathrm{~N}$-acetyl-L-cysteine inhibits primary human T cell responses at the dendritic cell level: association with NF-kappaB inhibition. J Immunol 162:25692574

10. Pahan K, Sheikh FG, Namboodiri AM, Singh I $1998 \mathrm{~N}$-acetyl cysteine inhibits induction of no production by endotoxin or cytokine stimulated rat peritoneal macrophages, C6 glial cells and astrocytes. Free Radic Biol Med 24:39-48

11. Song M, Kellum JA, Kaldas H, Fink MP 2004 Evidence that glutathione depletion is a mechanism responsible for the anti-inflammatory effects of ethyl pyruvate in cultured lipopolysaccharide-stimulated RAW 264.7 cells. J Pharmacol Exp Ther 308:307-316

12. Sekhon B, Sekhon C, Khan M, Patel SJ, Singh I, Singh AK $2003 N$-acetyl cysteine protects against injury in a rat model of focal cerebral ischemia. Brain Res 971:1-8

13. Victor VM, Rocha M, De la Fuente M $2003 \mathrm{~N}$-acetylcysteine protects mice from lethal endotoxemia by regulating the redox state of immune cells. Free Radic Res 37:919-929

14. Jatana M, Singh I, Singh AK, Jenkins D 2006 Combination of systemic hypothermia and $\mathrm{N}$-acetylcysteine attenuates hypoxic-ischemic brain injury in neonatal rats. Pediatr Res 59:684-689

15. Paintlia MK, Paintlia AS, Barbosa E, Singh I, Singh AK $2004 N$-acetylcysteine prevents endotoxin-induced degeneration of oligodendrocyte progenitors and hypomyelination in developing rat brain. J Neurosci Res 78:347-361

16. Paintlia AS, Paintlia MK, Singh AK, Stanislaus R, Gilg AG, Barbosa E, Singh I 2004 Regulation of gene expression associated with acute experimental autoimmune encephalomyelitis by Lovastatin. J Neurosci Res 77:63-81

17. Bell MJ, Hallenbeck JM, Gallo V 2004 Determining the fetal inflammatory response in an experimental model of intrauterine inflammation in rats. Pediatr Res 56:541546

18. Huleihel M, Amash A, Sapir O, Maor E, Levy S, Katz M, Dukler D, Myatt L, Holcberg G 2004 Lipopolysaccharide induces the expression of interleukin-1alpha distinctly in different compartments of term and preterm human placentae. Eur Cytokine Netw 15:30-36

19. Hagberg H, Mallard C, Jacobsson B 2005 Role of cytokines in preterm labour and brain injury. BJOG 112:16-18

20. Romero R, Sepulveda W, Kenney JS, Archer LE, Allison AC, Sehgal PB 1992 Interleukin 6 determination in the detection of microbial invasion of the amniotic cavity. Ciba Found Symp 167:205-220; discussion 220-223

21. Radi ZA, Kehrli ME Jr, Ackermann MR 2001 Cell adhesion molecules, leukocyte trafficking, and strategies to reduce leukocyte infiltration. J Vet Intern Med 15:516529

22. Esplin MS, Romero R, Chaiworapongsa T, Kim YM, Edwin S, Gomez R, Mazor M, Adashi EY 2005 Monocyte chemotactic protein-1 is increased in the amniotic fluid of women who deliver preterm in the presence or absence of intra-amniotic infection. J Matern Fetal Neonatal Med 17:365-373

23. Proost P, Wuyts A, van Damme J 1996 The role of chemokines in inflammation. Int J Clin Lab Res 26:211-223

24. Denison FC, Kelly RW, Calder AA, Riley SC 1998 Cytokine secretion by human fetal membranes, decidua and placenta at term. Hum Reprod 13:3560-3565

25. Callejas NA, Casado M, Bosca L, Martin-Sanz P 1999 Requirement of nuclear factor kappaB for the constitutive expression of nitric oxide synthase-2 and cyclooxygenase-2 in rat trophoblasts. J Cell Sci 112:3147-3155

26. Candelario-Jalil E, Gonzalez-Falcon A, Garcia-Cabrera M, Alvarez D, Al-Dalain S, Martinez G, Leon OS, Springer JE 2003 Assessment of the relative contribution of COX-1 and COX-2 isoforms to ischemia-induced oxidative damage and neurodegeneration following transient global cerebral ischemia. J Neurochem 86:545-555

27. Yamazaki K, Endo T, Kitajima Y, Manase K, Nagasawa K, Honnma H, Hayashi T, Kudo R, Saito T 2006 Elevation of both cyclooxygenase-2 and prostaglandin E2 receptor EP3 expressions in rat placenta after uterine artery ischemia-reperfusion. Placenta 27:395-401

28. Miyaura C, Inada M, Matsumoto C, Ohshiba T, Uozumi N, Shimizu T, Ito A 2003 An essential role of cytosolic phospholipase A2alpha in prostaglandin E2-mediated bone resorption associated with inflammation. J Exp Med 197:1303-1310

29. Perkins DJ, Kniss DA 1997 Tumor necrosis factor-alpha promotes sustained cyclooxygenase-2 expression: attenuation by dexamethasone and NSAIDs. Prostaglandins 54:727-743

30. Victor VM, De la Fuente M 2003 Immune cells redox state from mice with endotoxin-induced oxidative stress. Involvement of NF-kappaB. Free Radic Res 37:19-27

31. Biri A, Kavutcu M, Bozkurt N, Devrim E, Nurlu N, Durak I 2006 Investigation of free radical scavenging enzyme activities and lipid peroxidation in human placental tissues with miscarriage. J Soc Gynecol Investig 13:384-388

32. Cai Z, Pan ZL, Pang Y, Evans OB, Rhodes PG 2000 Cytokine induction in fetal rat brains and brain injury in neonatal rats after maternal lipopolysaccharide administration. Pediatr Res 47:64-72

33. Tsuji F, Miyake Y, Aono H, Kawashima Y, Mita S 1999 Effects of bucillamine and $\mathrm{N}$-acetyl-L-cysteine on cytokine production and collagen-induced arthritis (CIA). Clin Exp Immunol 115:26-31

34. Rahman A, Kefer J, Bando M, Niles WD, Malik AB 1998 E-selectin expression in human endothelial cells by TNF-alpha-induced oxidant generation and NF-kappaB activation. Am J Physiol 275:L533-L544 